\title{
Design Framework For Social Wearables
}

\author{
Ella Dagan, Elena Márquez Segura, Ferran Altarriba Bertran, \\ Miguel Flores, Robb Mitchell, Katherine Isbister
}

\author{
Computational Media Department, \\ University of California Santa Cruz \\ \{ella; faltarri; mflore21; \\ katherine.isbister\}@ucsc.edu
}

\author{
Department of Informatics \\ and Media, \\ Uppsala University \\ elena.marquez@im.uu.se
}

\author{
Design and Communication, \\ University of Southern \\ Denmark \\ robb@sdu.dk
}

\begin{abstract}
Wearables are integrated into many aspects of our lives, yet, we still need further guidance to develop devices that truly enhance in-person interactions, rather than detract from them by taking people's attention away from the moment and one another. The value of this paper is twofold: first, we present an annotated portfolio of 'social wearables', namely technology designs worn on the body that augment co-located interaction. The design work described can serve as inspiration for others. Then we propose a design framework for social wearables grounded in prior work, as well as our own design research, that can help designers to ideate by raising valuable questions to begin their inquiry with and use to evaluate their designs. We illustrate the evaluative value of this framework through two social wearable designs, each tested in the appropriate social setting.
\end{abstract}

\section{Author Keywords}

Design framework; social wearables; wearables; Research through Design; RtD; social affordances; co-located.

\section{CSS Concepts}

- Human-centered computing $\rightarrow$ Interaction design theory, concepts and paradigms.

\section{INTRODUCTION}

Wearable technologies, i.e. technologies integrated into our clothes, jewelry, and other accessories that people wear, fueled by advances in sensors and connected technologies, as well as increased computing capabilities of mobile and embedded technology [24], have been in the limelight for over a decade [87]. We refer to 'wearables' as including 'smart fashion' designs [76], and as their production techniques continue to advance $[17,56]$ "devices are going to become smaller and yet more powerful, enjoy greater connectivity and utilize more sophisticated input and output" [33].

Permission to make digital or hard copies of all or part of this work for personal or classroom use is granted without fee provided that copies are not made or distributed for profit or commercial advantage and that copies bear this notice and the full citation on the first page. Copyrights for components of this work owned by others than the author(s) must be honored. Abstracting with credit is permitted. To copy otherwise, or republish, to post on servers or to redistribute to lists, requires prior specific permission and/or a fee. Request permissions from Permissions@acm.org. DIS '19, June 23-28, 2019, San Diego, CA, USA

(C) 2019 Copyright is held by the owner/author(s). Publication rights licensed to ACM. ACM 978-1-4503-5850-7/19/06\$15.00

https://doi.org/10.1145/3322276.3322291
Currently, most commercial wearable designs still focus on personal data tracking and management for the sake of self improvement (e.g. Fitbit [30]), on healthcare support in helping to diagnose, monitor or offer treatment advice (e.g. [22], [66], [80]), or on networked communication (e.g. Apple Watch [86]), neglecting the support of interaction between people who are co-located.

Wearing anything on the body affects in-person social interaction on some level. Clothing and accessories have been part of the human experience for thousands of years, and are used not just for warmth and protection, but also, to signal to others about one's own identity and influence social interaction [11]. Thus, wearable technology is already playing a social role [85], even in the absence of explicit technological features aimed at augmenting copresence. As Don Norman points out, imbuing worn items with computation is an opportunity to further augment social experience, but also, an opportunity to wreak distraction and disconnection [67]. Some commercial experts say that the next phase of wearable technology will be "The Social Age" [81]. In this paper, we reflect upon what this social age of computational wearables could look like-how such devices could enhance social experiences, and even create new forms of social experience. At a fundamental level, we are interested in how computation might truly enhance the social aspects of co-located experience when included in worn objects and apparel.

To get at these questions, we have taken a multi-phase approach. First, we closely examined extant examples of socially-focused wearable devices drawn from research, art, and fashion contexts. We then used this portfolio of examples, along with a review of related theoretical and empirical work, and our own work, to create a design framework. The framework suggests illustrative figures and open questions to ponder when attempting to design future social wearables. It leaves room for interpretation, so designers have flexibility in crafting solutions to particular needs and contexts. We illustrate the potential evaluative power of this framework through using it to evaluate two social wearables from our own work. They stem from Research-through-Design (RtD) [37, 94, 95] approach and were developed concurrent to the creation of this framework, influencing and being influenced by it. Although these designs target different use contexts, both are aimed at a preferred state of social value [32, 94], 
enhancing co-presence, focusing people's attention on one another rather than on their devices, and providing opportunities for people to interact with each other through using technology, but not for the sake of using it.

\section{BACKGROUND AND RELATED WORK}

Humans are social by nature. Establishing good communication and social interactions is what allows us to thrive as a species [65]. Quality in-person interaction has clear health benefits [52, 92], is an important indicator for enhanced productivity in business settings [69], and also contributes to our happiness [5]. Prosocial behaviors such as communicating emotions, authenticity, reciprocity, cooperation or compassion are also highly valuable in the personal domain [8]. In our work we share a similar approach to the design agenda of Slow Technology, in that the focus of the designs are not on "efficiency in performance" [41], but rather on the quality of the unfolding social experience.

Regarding wearables, we learn from [49] what people value in general, e.g. "meaningful coupling of aesthetics with function;" and in specific kinds of wearables, e.g. kinetic wearables have the potential to afford "a fluid representation of self" [49]. Wearables as assistive technology is an established research domain (e.g., [7], [39], [57], [78], [79]). [33]'s vision for wearables is as "ubiquitous augmentative technology used by everyone." [4] focused on the application of wearables, what product types were used for which application and its distribution on the body surface. Others have studied the selection of on-body location for wearables [93]. Some study wearables in the context of skin interfaces and Cosmetic Computing $[18,50,51,54]$. Researchers have also evaluated on-body location for gestural interaction with devices. They found it important to address aesthetic expression in wearable technology as it relates to social needs (e.g. users might want to use "trouser pockets for more inconspicuous or naturalistic interactions with a textile-based wearable device") [23].

Most of this previous work taps into social aspects of wearables conceptually, from the wearer's perspective. The 'social' we focus on here is of encouraging and promoting co-located social interaction to facilitate prosocial behavior. This does not mean that all social wearable designs should adhere to being socially acceptable. We take an interactional approach to sociality and accompanying emotions [6], and take an experiential approach to technology design [43]. We approach our study of social wearables from the perspective of the wearer (personal) as well as from the perspective of others (social), and the relationship between the two. There are some HCI projects that align with these ideals that incorporate wearables [45, $46,61,62]$. From these, we have drawn concepts that have shaped both our framework and our design and development process. For example, social affordances reference prosocial behaviors that are supported by the design [47, 58]. In design research, e.g. [58] and [47], they have been used as guiding design concepts providing a fruitful level of abstraction similar to experiential qualities [55]: they capture design ideas explicitly referring to and evoking experiences, in our case social experiences; but they are concrete enough to relate these to properties of the technology, such as its functionality, interactivity and (technology) affordances [35]. This bridge between the abstract and the concrete is essential to the framework presented here: it is at the core of our analysis of areas of social wearables. We found the following social affordances articulated in previous works particularly relevant: social signaling ("supporting and augmenting the expressivity and readability of verbal and non-verbal cues") [58]; social appropriateness (signal the type of interaction considered acceptable); and spectator sensitivity ("facilitating spectatorship") [58]. Also, shaping of proxemics (the "use of sensors to shape the flow of interpersonal distance (proxemics) in prosocial ways") [47]; and Supporting individualized performances and social flexibility (enables "a broader spectrum of social encounters") [47].

\section{RESEARCH APPROACH}

We began by surveying over 50 wearable designs from research, industry, and the art world. We were searching for designs that focus on the co-located social space (excluding wearable assistive technology as we consider it to be a separate category). Three researchers worked individually to thematically analyze this collection by reading design descriptions, articles, press releases and reviews, and by watching promotional videos when available. We eliminated designs that were not intentionally affecting the co-located social space. We then created an annotated portfolio, highlighting "family resemblances," between designs [36, 55], which resulted in the social value areas introduced in the next section. These delineate existing and potential roles of social wearable designs, responding to the question of what computation might add to co-located social experience through the use of wearables. Towards creating a framework for social wearables, we discussed the designs within each area using concepts from previous works, such as social affordances. This led to theorizing on how these designs might work towards those ends, and why. The result was the formulation of fundamental design considerations in the form of guiding questions to ask when designing and evaluation social wearables. We found these questions useful for discussing results from past projects, and for driving ongoing projects, helping us identify technical considerations (e.g. sensing, actuating, shape, etc.), considering at the same time important social implications. We illustrate the evaluative potential of the framework by reporting on the design and study of two social wearables projects. They were created concurrent to the development of this framework, and both impacted and were impacted by it. 


\section{AREAS OF VALUE FOR SOCIAL WEARABLES}

Here we describe the two main areas that emerged from the survey of existing designs, framed by using an annotated portfolio approach $[36,55]$. We focused on examples that suggest what computation could add to the social aspects of co-located experience.

\section{Augmenting existing social signaling}

Computation can help one present them self to others as a person, and as a social being: who they are; their needs, desires, and preferences; what they mean; and how others can best interact with them. Much of this is already signaled or communicated through verbal and non-verbal means, as well as with clothing and other cosmetic choices. Computation can enhance these signals. It most commonly does this with some sort of extended visualization (light effects, color changes, kinetic movement) of social signs, at times reinforced with adding auditory signals. The designs in our portfolio helped us think about the following questions: How can computation enhance existing social signaling cues from the wearer? Is computation automatically reacting to, or intentionally being triggered by the wearer, or by others? How much control do the wearer and others have on activating it? Do others perceive and/or act upon the outcome or effects of computation? What does it add to, or change, in the social experience of both the wearer and others?

\section{Spectacle, Novelty and Drawing Attention to Oneself}

Wearables in performance arts (e.g. theater, dance, cosplay), and fashion have been used to characterize and present the wearer in a particular way to the audience, with the goal of impressing, surprising or delighting them. Computation adds an extra layer of novelty, surprise, and sensual pleasure. It is integrated into existing garments, or used to craft others from scratch, creating new affordances and infusing garments and accessories with new dynamic qualities [12, 34, 60, 71]. Some wearable designs in this category are works by renowned avant-garde fashion designer Hussein Chalayan [73], who has been experimenting with embedding computational technology in his runway pieces for two decades. Among his notable designs are the laser dress [16], or the creative use of motor systems and pulleys to change shape, style and even the presence of clothes [15]. Studio XO [91] designed the Bubelle dress [44] which changes colors based on the wearer's mood. They also designed Volantis [31], a dramatic showpieces. It is a flying dress for Lady Gaga, remotely controlled by 12 battery-powered propellers.

Attached, detached or independent moving ensembles have also been experimented with; Kao et al. introduced Kino, a robotic system accessory design that incorporates untethered motoric moving pieces to the garment. This design explored the potential of kinetic wearables to express personal style and fluid representation of self [49]. Similarly, The Butterfly Dress [29] integrates detached and reactive wings of a robotic butterfly. Using proximity sensors, the behavior of the wings changes depending on the presence and motion of co-located others. For performance's sake, they can also be activated remotely via cell phone.

\section{Extending Non-Verbal Social Signs}

Some designs use computational power to extend, or augment the aesthetics of existing, non-verbal communication tools. Several artful designs react to the social context or the wearer's emotional state by performing kinetic movements to reflect social signaling. Shiver $1 \& 2$ [48] are responsive luxurious feminine nightgowns that react when others touch them by activating embedded haptic motors. Shrug [9] reacts with perceptible movement - it is worn on the back as a cascading feather harness that ruffles when a wearer is stressed (measured through heartbeat sensors). Caress of the Gaze [28] is a 3D printed, poncho-like wearable that uses computer vision to detect when people look at it, reacting by shape-changing thanks to shape memory alloy actuators. Other designs use servo motors for dramatic movement effects. Monarch [42] is a shoulder harness made of textile forms that "expands and contracts in response to the movement of the wearer's muscles" [63]. The activation of Monarch is intentional: the design requires the wearer to considerably engage their arm muscles to process a state change and react. It is designed to physically extend expressive body language signs and better indicate the wearer's “enthusiasm, excitement, assertion or aggression, flirtation or mischievousness" [63].

Expressive Wearable [27] is a hat with small fans that fold and unfold when the wearer is experiencing a loud noise, a bright light or a body coming too close. The hat's movements resemble the bodily gestures people might make when feeling uncomfortable; covering one's ears when hearing loud noise, covering one's face when embarrassed. Even more dramatic are the gestures of the Robotic Spider Dress [77], a 3D printed dress with mechanical spider legs that both expresses the wearer's emotions (as measured through various biometric), and also reacts to movements around the wearer, in an intention to "protect their personal space" [77]. A few other designs address the notion of signaling to others the appropriate social or personal distance. Space Dress [83] inflates based on the wearer's request. Another design, part of the Urban Armour collection [20], is a skirt that uses servo motors to spread in an umbrella-like shape when someone comes too close to the wearer (measured through proximity sensors). Finally, Barking Mad [3] is a coat design that also uses proximity sensors to "respond to infringements on personal space with the sound of barking dogs [3].

\section{Intervening in the Social Situation Proactively}

We found a few examples of designs that proactively intervene in social situations, supporting wearers and nonwearers to interact with one another in novel ways. These designs usually include more than one device, and many create a clear and actionable call for interaction, which may 
support connection and bring people closer together. This could be manifested through suggesting a social activity such as a playful interaction, a task-oriented interaction, a collaborative creation, or any other activity that requires interaction between people. These designs helped us think about the following questions: How can computation be used to get people to interact with each other? What is the activation mechanism? How does the design entice or reward social interaction? How many people are they affecting? How many are wearing designs or parts of the design? Interestingly, most of the examples we found focus on supporting two-people interaction.

For example, the Social Body Lab [82] created the SuperHero Communicator Cuffs [21], a DIY instruction kit to make a pair of cuffs that can send and receive an alert signal from a remote partner in times of need. The cuffs are controlled by a specific hand gesture that triggers an electronic switch, which sends and receives wireless signals. They also made The Nudgeables Accessory Kit [68], a "modular hardware kit for creating paired sets of wireless wearables accessories" [68]. While partners are in the company of a larger group of people, they can use it to send and receive secret nudges from a distance. Its design flexibility lets users customize the sensor (choose what triggers the signal) and actuator (the form of the signal e.g., vibration, motor, LED, light etc.) as they wish. They can also embed the Nudgeable technology into a pre-made accessory of their choice (e.g. a tie, a scarf, pendants, or socks).

Some designs in this space proactively try to encourage people to physically come close and touch each other through the use of closed circuits. Hug Jackets [70] consists of a pair of jackets with conductive fabric applique on both front sides. When the wearers hug, they close a circuit that triggers lights at the back of both jackets. This also activates a heartbeat sound that increases its pace accordingly to the duration of the hug. The Co-dependent Gloves [10] are a pair of gloves, each worn by a different person. When the two hold hands they close a circuit and the gloves begin to warm up. Also promoting social touch, Hotaru [1] is a game which uses controllers that encourages players to hold hands and perform synchronized gestures. One player wears a back tank and a glove, and the other a glove and a gauntlet. The first player needs to collect energy making hands movements "in an energy collecting gesture" [45]. This energy is displayed through lights on that player's back tank. It can then be released by holding hands with the second player, who then performs a quick upward gesture with the arm on which they wear the gauntlet, causing a sound indicating that the energy has been released. This design creates interdependence between players, a design quality that "requires shared attention and mutual awareness" and "encourages and rewards co-located interaction" [45]: the wearer of the tank cannot see their tank's energy level, hence they have to depend on their partner to know when it is filled. Likewise, both players need to hold hands to 'transfer' the energy. A final example of a wearable that encourages people to come close and physically touch is Massage Me [59]. This alternative video game controller takes the form of a jacket/vest that requires two people, the wearer and the player. The player controls the game by pressing on specific points on the vest of the wearer. The wearer receives a light or deeper massage, depending on the player's excess of energy.

\section{SOCIAL WEARABLES DESIGN FRAMEWORK}

Here we present the framework that emerged from our the study of the wearables in the portfolio. Through analysis of this collection of designs, we found that computation can augment cues that people use to present themselves as individuals and social beings, invite social interaction, bring people together, and support physical and emotional closeness. Here we focus on the questions of how this is done, and why it would work. We present our framework in the form of a series of guiding questions, that we formulated as we sorted through extant work. These questions were shaped by concepts in prior work, such as social affordances, as well as a constant consideration of both the wearer and others (wearers or non-wearers). Some of these questions were technical (e.g. what does the wearables sense? what is the output?); others specifically focused on the control of the devices and the roles of those involved (who or what controls the device? are co-present people aware of this? does it involve intentional action from the wearer or others?); some focused on demands or requirements on those co-present (e.g. what commitment is required in terms of attention? proximity?); some were about the impact on the social experience (e.g. does it bring people together? does it initiate or encourage new social interactions? does it support people to be better together with others?). In the following, we present these deeply interconnected questions; the first questions constitute the building blocks for the latter more abstract considerations.

\section{Sensing}

How is the device activated? What is being sensed? What means or gestures required? Does activating the device happen automatically or require people's intentional input? Who controls the device activation and who controls its deactivation? Is it the wearer, the wearer with others, or others alone? In the surveyed examples we found recurring methods of activation based on the sensor mechanism, which could be manual or completely automatic. This led to considerations regarding the intentionality of wearers and non-wearers to trigger the wearables (See Fig. 1). We understand intentionality here as deliberate activation of the wearables, which is not always straightforward when analyzing the wearable designs. For example, designs triggered by proxemics work automatically, but they could be intentionally activated by both wearer and others, through coming closer. Other examples could be understood differently from the perspective of the wearer and that of others. 


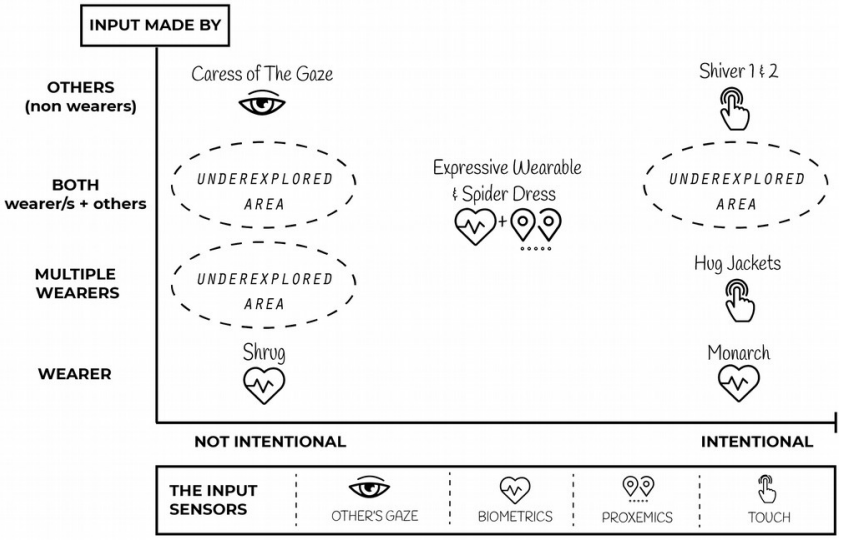

Figure 1. Matrix of examples depicting who is responsible for triggering the input, the degree of their intentionality, the input sensors, and the underexplored areas. Icons credit: Noun Project \{icongeek; iconsphere; Marek Polakovic; Ramesha\}.

For example, designs requiring social touch (e.g., Shiver 1 \& 2 [48]) could present scenarios of non-intentionality by the wearer, and intentionality by others; or cases in which social touch happens casually or by accident, triggering the device without intention. Hence the classification of wearables in Fig. 1 is only illustrative; the intentionally of those involved can only be established by themselves; yet thinking about automaticity in sensors and how these can be affected by human intentionality can be useful when designing to impact a co-located social scene. Additionally, some of the examples illustrate unconventional and inspiring uses of sensors regarding intentionality. For example, Monarch [42] uses muscle contractions. Humans contract muscles both intentionally and unintentionally, yet this device explicitly targets the former through sensors with, we assume, a small trigger sensitivity requiring intentional effort by the wearer. Finally, sensing mechanisms speak of social affordances, which can have an important social impact. For example, devices that sense proxemics have potential for a shaping of proxemics affordance [47], encouraging co-present people to move closer to one another. Wearables activated by touch (e.g. [2], [10], [70]) show promise to bring people intimately close, which can enhance existing social bonds [72], help create new ones increase compliance and liking of another person $[40,64,89]$, and help people reflect their emotions.

\section{Actuating}

What is the output? Is it noticeable or not? By the wearer? By others? Through which means (e.g. visual, auditory, tactile)? Is it actuating in real time or is it delayed from the sensing? This talks about the performativity of the device and its impact in a social context. Concealing the actuation of wearables can help in situations where the wearer does not want to attract attention. However that kind of actuation would not be effective to augment existing social signaling. On the contrary, these designs work because they are highly expressive. For example, the actuation of the Robotic
Spider Dress [77] is obvious to both wearer and others colocated people around. We found that expressive wearables are common in the area of proactive social interventions.

The sensory channel through which actuation manifests also impacts the wearable's performativity. E.g. while heat will likely be only noticed by the wearer (and possible those in physical contact with them); a visual output is more likely to be perceived by others (depending of course on matters of size, and distance of others). Also among competing channels, the visual will likely capture most attention, given that vision is our primary sense [90] and people are highly used to interpreting diverse types of visual cues (e.g. movement, shape, light) [38].

\section{Sensing-Actuating Interplay}

What is the interplay between sensing and actuating? How transparent is this to the wearer and others? This block builds on the previous two, focusing on the relation between input and output and who has access to this information. It concerns aspects of performativity, awareness, and control. If the wearer is being sensed, do others notice this? Do they have access to the output? Interestingly, the area of sensing others (or both the wearer and others) for an output exclusively accessed by the wearer is underexplored. The transparency of the functioning of the device concerns whether the mapping between sensor and actuator can be deciphered. For this, we borrow Reeves et al.'s [72] classification of public interfaces regarding the visibility of manipulations (input) and effects (output) (See Fig. 2). Wearables are secretive if both input and output are concealed; expressive if both are revealed; magical if the input is concealed, but the output is revealed; and suspenseful if the input is revealed, but not the output. We can think about all these considering both the wearer and others. For non-wearers of the Robotic Spider Dress [77] coming closer to the wearer, and who are unaware of its functioning, the wearable will likely feel magical. What triggered that device? Why is it moving? For the wearer, the dress is expressive. Interestingly, we did not find many examples of secretive or magical wearables, which are interesting future areas of exploration. Having a sense of interplay between input and output can impact intentionality. If a non-wearer of the Robotic Spider Dress [77] is aware of its functioning, they can consciously trigger the wearable by walking towards the wearer. This speaks of affordances of prosocial behavior.

\section{Sensing-Actuating Interplay between Wearables}

How many devices are there? What is the interplay between them? Here we look at several wearables, identical or not, and how they affect and are affected by one another. Here symmetry speaks about two wearables whose input and/or output are identical and have an identical impact. Interdependency refers to whether one device needs the other, or another person's action to function. For example, the Hug Jackets [70] are two identical wearables that respond when they come in contact with each other. They 
are symmetrical and interdependent. Hotaru [45] is designed to create interdependency between players, with two different wearables. They have a certain symmetry, since they sense one another, but the person with the tank does not see it while the other does; and the person with the gauntlet can release energy, which the other cannot.

All the examples we found that implement some form of interplay belong to the area of Intervening in the Social Situation Proactively, which is not surprising. Designing interplay between wearables will likely bring people together (e.g. [70] and act in synchrony (e.g. [1])). We have found that group interplay is an underexplored area. What effect would a symmetric and interdependent group of wearables cause among their wearers? It could possibly have similar intimate effects to those for two people, but for a group (e.g. a family). What about an asymmetric and interdependent group of wearables? These could be used to send social signals to one particular person (e.g. students to the teacher).

\section{Personal and Social Requirements}

What are the personal and social commitments that the wearables require? E.g. what degree of focused attention is required from the wearer and others? We consider this as a spectrum where low commitment is represented by wearables that do not require intentional effort from the wearer to activate and use them, such as automatic tracking of biometrics, gestures, or environmental changes which are outside of the wearer's control (e.g. Shrug [9], Barking Mad [3]). They do not necessarily detract from engaging in other activities or interacting with others. Medium commitment represents wearables that require some degree of intentional effort to activate, but do not completely hijack the wearer and other's attention and energy. Interacting with these devices could be parallelized with other activities. These typically use inputs that can be "provided" while engaged in those other activities (e.g. Co-dependent Gloves [10]). Last, high commitment represents wearables that completely require of the interactants attention, orientation, and energy, likely preventing them from engaging in other activities (e.g. the Hug Jackets [70]).

These typically require concrete and specific gestures. Devices that require high levels of commitment to activate or interact with them could unwantedly detract from the inthe-moment co-located social experience. However, if high commitments such as focused attention are required by both wearer and others, or by both wearers, the wearables might foster a joint activity that supports how the interactants relate.

Does the device operate in a way that is respectful of people's time and attention? The question of commitment leads to this one. In the Guide to Anticipating The Future Impact of Today's Technology designers are suggested to ask "Does the business model behind your chosen technology benefit from maximizing user attention and engagement - i.e., the more, the better?

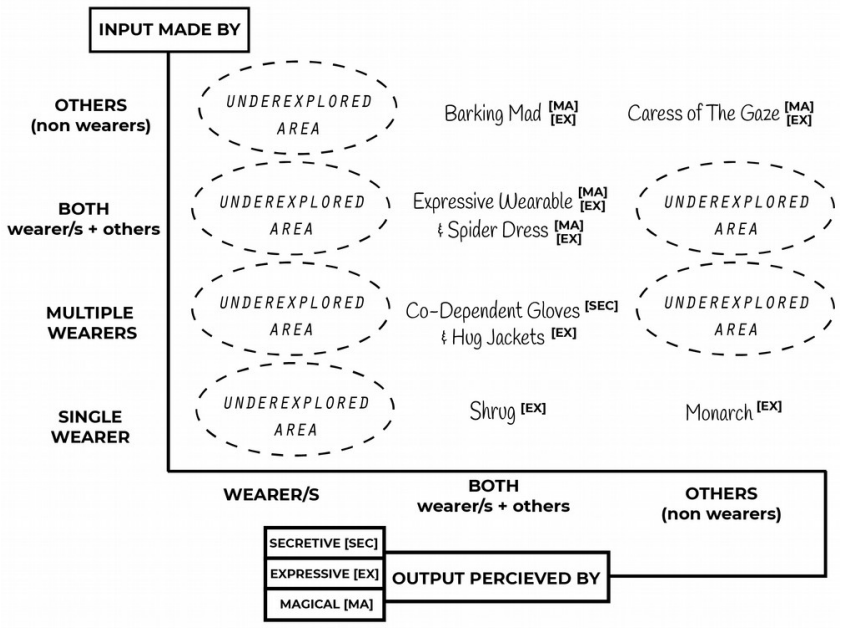

Figure 2. Matrix visualizing who "is sensed" and who "senses" the wearable, (i.e. can perceive its output), the kind of output (expressive, secretive or magical), and underexplored areas.

If so, is that good for the mental, physical, or social health of the people who use it? What might not be good about it?" [25]. Tristan Harris, formerly Google's chief ethicist, made a call to tech companies to encourage "time well spent," encouraging designers to consider how their design may steal time from other activities or from just being with other people. Devices should not demand people's time and attention for reasons that do not benefit them as users, if those devices are designed to be used in everyday life. However, for those engaged in critical design, demanding (hard to ignore) or disrespectful devices could be intentionally designed to draw attention to particular elements people take for granted, or usually try to ignore. When designing social wearables, it is important to be aware of the amount of investment (of time and attention) the devices requires from the wearer and others.

\section{Social Acceptability}

In what social environment the interaction occurs? Some designs might be worn during a particular situation (e.g., Massage Me [59] would be when people meet to play), but others might be designed to be worn all day (e.g., Nudgeables [68] could be worn all day around the office). In considering the following guiding questions, it is key to bear in mind that what users might find acceptable in one setting might not work somewhere else.

Is wearing the device considered socially acceptable? Are the sensing/activation and actuation of the device socially acceptable? What is and what is not appropriate changes over time. Before handsfree headsets people who talked to themselves, with no one by them, were perceived as potentially insane; Nowadays it would not raise any eyebrows [74]. The input required to activate a device, as well as its output and how it is perceived could greatly affect how comfortable people would feel in a particular social environment: "Uncomfortable social situations can also arise from the gestures and touches users make with 
wearable technology to interact and control devices" [93]. Dunne et al. [23] found in a case study of on-body gestural input that people wanted to avoid "social discomfort," they wanted to look natural, blend in and not make obviously awkward gestures. Dunne et al. suggest that on-body interactions could rather leverage existing interaction with clothing to design more secretive and more acceptable wearables. However they also mentioned that "cool aspects of wearing the device, and the novelty of the interaction" were considered "positive aspect encouraging adoption" [23].

Are the sensing and/or actuation methods customizable by the wearer? Empowering the wearer to customise their wearable and adapt it to their social situation does not only allow expressivity, but it might also support prosocial behavior, and increase acceptability. From all reviewed designs, The Nudgeables Accessory Kit [68] was the only one affording customization.

What is the best on-body location for the wearable to bring people together? [93] mapped the areas that should be considerably agreeable for on-body interactions. Here we focus more on the social and emotional impact that interacting with devices worn in particular body areas has for the wearer and others. Designing wearables that make use of socially acceptable body areas of interaction can work for bringing people together without requiring too much intimate contact (considering the complex sociocultural context, e.g., the acceptability of men and women in regards to touching in different cultures). On the other hand, we have seen with designs like [45] that relevant output or interactable parts in unusual areas where the wearer does not have access to (e.g. the back) can encourage, reinforce, or establish interesting social connection. However, precisely because those areas are not as accessible for the wearer, we encourage designers to consider implementing measures to give both the wearer and others a choice about if and how they interact.

It should be noted that some of the more artistic approaches to wearables might deliberately design for low social acceptability in order to provoke reflection and debate. Although these kinds of designs (e.g. [3], [77]) may seem unlikely to promote prosocial behavior, we included them in our discussion of social wearables because they embody some usefully extreme qualities of the design space.

\section{USING THE FRAMEWORK TO EVALUATE DESIGNS}

Intervening in the Social Situation Proactively was found to be an underexplored area in our portfolio, yet examples there show much promise in terms of facilitating interesting co-located social experiences. In this section, we present two social wearables that populate that area. They were designed to heighten existing social signal processes, and create and encourage new social interactions. Here, we briefly introduce these projects and use our framework as a set of lenses to explain and evaluate design decisions.

\section{Lågom}

We designed the Lågom social wearable [14] to support group discussions that welcome balanced verbal participation from group members (e.g. brainstorming sessions). At times, striking a balance in participation is challenging when there are proactive members and also avoidant or shy people. Providing peripheral visual feedback on one's participation can help participants balance their contributions, especially on the "over and under participation" ends [19]. Several designs leverage participation feedback via peripheral vision [19, 53, 84]. Despite usefulness for balance, using peripheral vision during a discussion requires gaze commitments that can be distracting for those involved. Lågom (Fig. 3) is a playful flower/plant-like looking wearable ${ }^{1}$ that provides haptic feedback to the wearer as it senses their voice, as well as visual feedback to co-located others to increase the group's awareness of self and others' participation to support more balanced group discussions. Its name comes from a Swedish word with no direct English translation, conveying a long-lived Swedish ideal of optimal amount, "not too much and not too little" [88]. We iterated the prototype to explore various feedback modalities, vibration patterns, and input sensibility. These were tested during in-lab group discussions by lab members and invited guests. Multiples of the last prototype were deployed and studied in a (semi-) natural setting: a graduate class where nine students discuss research papers. Through observations, semi-structured interviews, and a survey, we compiled the users' opinions about Lågom, and studied its potential to increase awareness of, and to impact, participation.

\section{Sensing}

Lågom is worn by all members of a discussion group. It senses the wearer's voice automatically once it is turned on (not intentional), but the wearer needs to intentionally signal (i.e. slide a switch) the beginning and the end of the discussion period (by pressing buttons). Users must synchronize their intentional signaling of the discussion period for the Lågoms to calculate a fair contribution assessment. This joint action is envisioned as a communal effort to support equality during the discussion.

\section{Actuating}

Lågom has an expressive in-the-moment feedback output for others, and a secretive one for the wearer. To support awareness of ongoing verbal contributions, haptic feedback is provided to the wearer through pulses that increment their frequency with continued verbal participation (and decrement or cease once the wearer stops talking). Inspired by the social affordance of spectator sensitivity [58], others are provided with visual feedback through 10 LEDs that light up depending on duration of participation. Another expressive visual feedback was implemented for wearers and non-wearers showing cumulative participation of the wearer using the LEDs. They would be triggered through

1 Here, a video description of the device: https://youtu.be/RRv0wEOLaaY 
buttons on the interface at the end of the session, allowing participants to compare and discuss their overall participation if they wished to do so.

The study showed that the wearables worked well to increase the awareness of participation of all users, changing the habitual participation of some of them. Users who usually tend to shy away from participating actively in group discussion reported they found the design useful: it helped them be more aware of their participation, and that in turn encouraged them to put more effort into making their voices heard. Participants had varied appreciation of the feedback modalities. The haptic feedback elicited strong reactions: A usually participative participant, disliked it and interpreted it as a sign to stop talking. Another participant really liked the haptic feedback, and commented that it invited her to participate. A third participant did not feel comfortable with this sort of feedback and decided to participate in the discussion only using their body gestures. A fourth participant found the haptic feedback distracting, but imagined getting used to it. This comes to show that when designing for people it is important to test with actual users.

\section{Sensing-Actuating Interplay}

While the in-the-moment haptic "nudges" are secretive, they are mapped to the LEDs on the front of the interface which are expressive as an output. A device does not require others' devices to function, but we envisioned it would be used together with others. These wearables are identical (in terms of functions) and symmetrical. The only interdependency comes from the need to synchronize them at the beginning and end of the discussion to be able to compare overall participation.

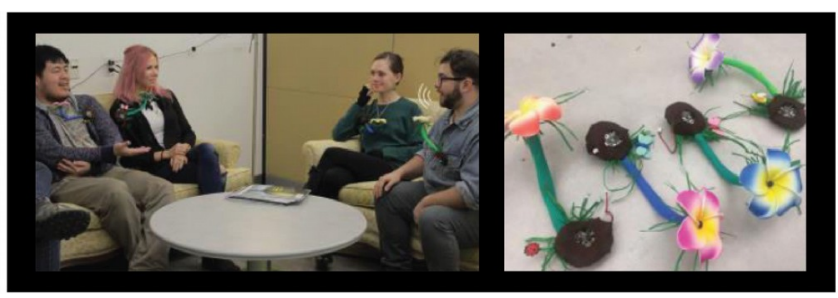

Figure 3. Left: Lågom is used by all members of the group discussion. Right: the Lågom design.

\section{Personal and Social Requirements}

Lågom requires a low commitment from the wearer and others to operate. The wearer is meant to talk freely, although due to limitations of the microphone we asked participants to not turn their heads away while talking. Others can visualize the speaker's participation duration through the LEDs, which can draw their attention. However, they are worn on the lapel and visible while maintaining eye contact with the speaker. We sought that the lights be visible but not distracting, and help nonspeakers focus on the speaker. Seven out of nine participants reported their attention was not actively focused on the LEDs and did not really notice the light changes. Two mentioned the LEDs drew their attention. They distracted one of them from the discussion, but helped another one to be more focused on the speaker. Some suggested the design would attract less attention if it was smaller. One wearer associated the vibration behavior to that of their phones, and other smart devices, which triggered too strong of an alert response.

\section{Social Acceptability}

Although a device is not dependent on other people, we designed Lågom to be worn by all discussants. We considered this may increase its acceptability. For that same purpose, we infused a playful aesthetics to the design (inspired in water-squirting flowers used by clowns), and considered it to be gender neutral (all genders can wear flowers). The looks of the device was liked by most participants, and was considered 'cute'. However, a few participants brought up the flower shape and suggested it gave the design a feminine look, and said they prefer it to be more neutral looking. The on-body location was also discussed in regards to acceptability; a user commented her flower was attached too close to her chest, which felt somehow awkward and distracting.

\section{True Colors Wearable}

We designed the True Colors [13] social wearable to heighten existing social signaling processes and encourage social interactions between wearers and non-wearers. They were designed for a group of players in a sci-fi Larp (live action roleplaying) event: New $G y r^{2}$. Similarly to previous work [58], we included the designers of the Larp, the Event Horizon [26] team, as co-designers during a three months design process involving multiple iterations and testing of the prototypes in diverse settings. True Colors is Y-shaped, worn around the upper chest area, shoulders and upper back (see Fig. 4 and video $^{3}$ ). It has a variety of functions, each targeting a different kind of social purpose: The stunning function was designed to express an attack behavior to others using LEDs and sound effects; the true color, an affiliation color each device had, displayed through LEDs; the affiliation function, which allowed players to display or disguise their true color; the overload function signaled both to the wearer and to others the moments of the wearer's breakdown - in which the wearer was "incapacitated"- through flashing red lights and sound effects; the healing function allowed others to mitigate the effects of overloads through social touch, triggering a rainbow light effect. Breakdowns and Healings could be provoked through hacking the wearable, by inputting a code in a keypad at the back of the device. We deployed 13 wearables during the three-day Larp event $(\sim 32 \mathrm{hr}$ of gameplay), and where 109 players participated. We documented the event through on-site observations, semistructured interviews, and a questionnaire focused on general impressions of the device, and interesting

2 More information: http://www.eventhorizonlarp.com/2-new-gyr.html 3 Here, a video description of the device: https://tinyurl.com/y6xbw813 
interactions with it, and with others as a result of the device. In the following, we use most repeated answers to assess each design aspect of our devices.

\section{Sensing}

Overall, the front part of True Colors was designed to be intentionally manipulated by the wearer (affiliation color, stun, overloads); while the back was designed to be intentionally manipulated by others (healing, overloads), hence through shaping of proxemics affordance [47]. Wearers could intentionally change their affiliation display by touching the capacitive sensors on the front right side; and trigger a stun by pressing down on the front left side for three seconds. They could also intentionally trigger an overload to create a breakdown scene for them and colocated others.

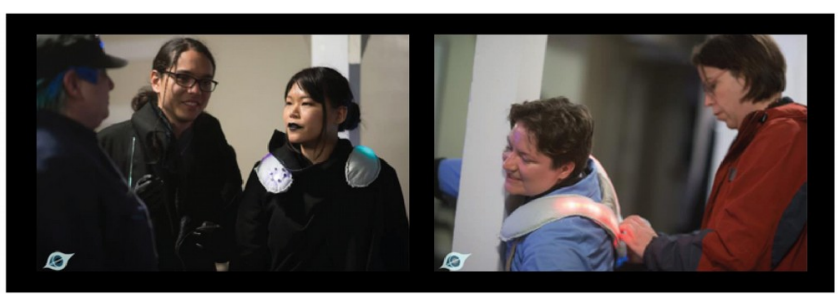

Figure 4. Left: The player on the right is wearing True Colors. Right: a non-wearer healing a wearer's overload. Images Credit: (c) Event Horizon.

Others could intentionally help heal wearers in an overload through social touch, i.e. a capacitive sensor pad at the back of the device. Special players could intentionally inflict an overload or completely heal through inputting a special code on the keypad at the back. Importantly, we designed overloads to be automatically and periodically triggered by a pre-programmed timer. This was meant to give players a sense of lack of control over flaws of the wearers' devices.

\section{Actuating}

True Colors had two expressive outputs: sound and lights through LEDs, which varied for the different functions. Overloads displayed flashing red color lights and sound effects (see Fig. 4). For wearers, these afforded to experience emotional resonance [58] with their characters, helping them roleplay those scenes of breakdown, having "big feels" [13] and enhancing immersion. This expressive outputs, together with the wearer's roleplay enactment served as a strong call for interaction from co-present others, through strong social signaling affordances. When others interacted with the social touch pad at the back, the overload lights changed to a pulsating rainbow pattern, providing a pleasant feedback for the wearer and the helper, perhaps reinforcing the helping behavior, and further enhancing their emotional resonance [58].

\section{Sensing-Actuating Interplay}

Almost all the functions were expressive, except the true color function, which could be secretive (non-disclosed to others). Overloads were described by players as being "unpredictable" and "random," and having "perfect timing." We relate these comments to the fact that players did not trigger them themselves; they let the device automatically trigger them. This created a sense of vulnerability towards these breakdowns that were appreciated by wearers and non-wearers alike. The 13 True Colors devices we developed were almost identical. They varied in quality (i.e. frequency of automatic overloads), and "true color," the real affiliation color of the wearer, which the wearer could choose to display or hide (by displaying a different color). These modifications were requested by our Larp designers to fit each character's backstory. The devices were symmetrical, and not dependent on each other. However, they could create relationships of dependency between players: non-wearers (or other wearers) could help prevent overloads, and help wearers through them. Players embraced this voluntary interdependence greatly, and reported they created intimate encounters, and helped develop close relationships among players. Overloads were talked about in terms of vulnerability, which they overcame together. Players enjoyed asking for and receiving care: one responded that his device "helped [him] feel important and special and [...] initiate contact with other players" [13]. The wearable encouraged caring for others, one participant commented it "allowed me to express compassion" [13]. From a spectator perspective, watching others being cared for was noted as a very valuable emotional experience: "seeing [wearers] being soothed by friends was really touching" [13], which speaks of the spectator sensitivity and emotional resonance of the device [58].

\section{Personal and Social Requirements}

By design (or rather implementation), True Colors did not require high commitments from the wearer or others, i.e. most of the functionalities were optional - that is, players could choose whether to use them or not. Even if they triggered functions, most of them only involved tapping or pressing buttons (i.e. low commitments). However, overloads were out of the wearer's control (when triggered by the timer) and the game's rules established that they had to be roleplayed as painful moments, which required much effort and exclusive attention from the wearer. Interestingly, they triggered high social commitment responses; co-present others readily jumped in to help, or asked others to help the wearer. This happened even despite in-game animosities, which surprised players and us alike. Although healing and generally helping in overloads took remarkable effort and commitment from others, we found both wearers and non-wearers deeply enjoyed this experience.

\section{Social Acceptability}

True Colors is a wearable made to be worn during the New Gyr Larp by a particular group of players. Within the Larp world, wearing the device was not only completely acceptable, but part of what these players' characters were. The back of the device, meant to entice interaction from 
non-wearers, was carefully designed to signal where it was appropriate to interact, i.e. through a demarcated area at the back, which is considered socially acceptable to touch in public [93]. In addition, prior to the Larp, wearers and nonwearers were informed about this device, and its functionality and role within the Larp. Wearers chose to participate in our study and wear the True Colors. Hence players commented how they understood social touch was a pre-agreed interaction. However, it could be socially negotiated through game techniques that players rehearsed prior to Larp, meant to help others modulate and adapt the way they interact with each other.

\section{DISCUSSION}

Through the overview of both the Lågom and the True Colors social wearables projects, we demonstrate the value of our design framework in helping to shape the design of social wearables. Asking questions raised by the framework helped to keep our designs focused on augmenting inperson interactions strategically and impactfully. The framework also helped us to better evaluate and articulate the observed value of the devices from user tests in the target environments. The presented annotated portfolio can be inspiring and contributes to "mid-level knowledge" [55] in the field of social wearables. It offers a valuable resource to design researchers and student alike in pointing to the variety of wearable technologies outside those that are commercially mainstream. Our framework adds to it by raising questions that can potentially help guide and evoke deeper thinking on the topic. These questions are worth considering when designing wearables to be used in colocated social spaces, and illustrate how this has been done in previous works. It also reveals current works that show the social value wearables can bring, as well as underexplored areas. Our framework is motivated by the premise that it is important to think about wearables from a prosocial perspective; how these mediate, or interfere with social encounters, as they are part of people's social context.

Our goal is to inspire the next generation of wearables rather than answer the questions raised in the framework or use them to evaluate what should be considered to be a "good" social wearable. We created the framework to invite critical thinking about the future design of social wearables and offer a reflection on the current design space. Finally, we propose that other designers of wearables intended to support co-located social interaction can use the framework in similar ways to help shape and support their design and development process. We hope to encourage designers to explore the area of sensing others with an output that would be exclusively accessed by the wearer, or group interplay, as these are underexplored.

\section{CONCLUSION}

In this paper, we have reviewed the state of the art in social wearables, and synthesized a design framework through close analysis of these exemplar systems. We articulated the framework in the paper using these systems as examples, and then showed how it can be used, through a brief overview of two social wearables projects that our research team created and evaluated. We propose that this framework can be of value in helping other wearables researchers and practitioners to adequately consider the nuances of supporting rich co-located social interaction with appropriate design decisions.

\section{REFERENCES}

[1] Kaho Abe and Katherine Isbister. 2016. Hotaru: The Lightning Bug Game. In Proceedings of the 2016 CHI Conference Extended Abstracts on Human Factors in Computing Systems (CHI EA '16), 277-280. https://doi.org/10.1145/2851581.2889472

[2] Leonardo Angelini, Maurizio Caon, Denis Lalanne, Omar Abou Khaled, and Elena Mugellini. 2014. Hugginess: Encouraging Interpersonal Touch Through Smart Clothes. In Proceedings of the 2014 ACM International Symposium on Wearable Computers: Adjunct Program (ISWC '14 Adjunct), 155-162. https://doi.org/10.1145/2641248.2641356

[3] Barking Mad with Jordan Benwick | Emily Carr University. Retrieved June 28, 2018 from https://www.connect.ecuad.ca/people/work/27474

[4] Mary Ellen Berglund, Julia Duvall, and Lucy E Dunne. 2016. A Survey of the Historical Scope and Current Trends of Wearable Technology Applications. In Proceedings of the 2016 ACM International Symposium on Wearable Computers (ISWC '16), 4043. https://doi.org/10.1145/2971763.2971796

[5] Diane S. Berry and Jane Sherman Hansen. 1996. Positive affect, negative affect, and social interaction. Journal of Personality and Social Psychology 71, 4: 796-809. https://doi.org/10.1037/0022-3514.71.4.796

[6] Kirsten Boehner, Rogério DePaula, Paul Dourish, and Phoebe Sengers. 2005. Affect: From Information to Interaction. In Proceedings of the 4th Decennial Conference on Critical Computing: Between Sense and Sensibility (CC '05), 59-68. https://doi.org/10.1145/1094562.1094570

[7] LouAnne E. Boyd, Alejandro Rangel, Helen Tomimbang, Andrea Conejo-Toledo, Kanika Patel, Monica Tentori, and Gillian R. Hayes. 2016. SayWAT: Augmenting Face-to-Face Conversations for Adults with Autism. In Proceedings of the 2016 CHI Conference on Human Factors in Computing Systems (CHI '16), 4872-4883. https://doi.org/10.1145/2858036.2858215

[8] Brené Brown. 2015. Daring Greatly: How the Courage to Be Vulnerable Transforms the Way We 
Live, Love, Parent, and Lead. Avery.

[9] 2016. Coded Couture features clothes that detect lies and read tweets. Dezeen. Retrieved August 30, 2018 from https://www.dezeen.com/2016/03/17/codedcouture-fashion-futuristic-exhibition-pratt-gallerynew-york/

[10] 2009. Co-Dependent Gloves. Joprints. Retrieved January 2, 2018 from https://joprints.wordpress.com/2009/09/13/codependent-gloves/

[11] Diana Crane. 2000. Fashion and Its Social Agendas: Class, Gender, and Identity in Clothing. University of Chicago Press, Chicago.

[12] CUTECIRCUIT. CUTECIRCUIT. Retrieved August 30, 2018 from https://cutecircuit.com/

[13] Ella Dagan, Elena Márquez Segura, Ferran Altarriba Bertran, Miguel Flores, and Katherine Isbister. 2019. Designing 'True Colors': A Social Wearable that Affords Vulnerability. In Proceedings of the 2019 CHI Conference on Human Factors in Computing Systems (CHI '19), Forthcoming. https://doi.org/10.1145/3290605.3300263

[14] Ella Dagan, Elena Márquez Segura, Miguel Flores, and Katherine Isbister. 2018. "Not Too Much, Not Too Little" Wearables For Group Discussions. In Extended Abstracts of the 2018 CHI Conference on Human Factors in Computing Systems (CHI EA '18), LBW129:1-LBW129:6.

https://doi.org/10.1145/3170427.3188500

[15] Dazed. 2013. Inner Space: Hussein Chalayan. Dazed. Retrieved August 29, 2018 from http://www.dazeddigital.com/fashion/article/16705/1/i nner-space-hussein-chalayan

[16] 2012. Designed in Hackney: laser dresses by Hussein Chalayan for Swarovski. Dezeen. Retrieved August 29, 2018 from https://www.dezeen.com/2012/05/18/designed-inhackney-laser-dresses-by-hussein-chalayan-forswarovski/

[17] Tilak Dias. 2015. Electronic Textiles: Smart Fabrics and Wearable Technology. Woodhead Publishing.

[18] Christine Dierk, Sarah Sterman, Molly Jane Pearce Nicholas, and Eric Paulos. 2018. HäirIÖ: Human Hair As Interactive Material. In Proceedings of the Twelfth International Conference on Tangible, Embedded, and Embodied Interaction (TEI '18), 148-157. https:// doi.org/10.1145/3173225.3173232

[19] Joan Morris DiMicco, Anna Pandolfo, and Walter Bender. 2004. Influencing Group Participation with a Shared Display. In Proceedings of the 2004 ACM Conference on Computer Supported Cooperative
Work (CSCW'04), 614-623. https://doi.org/10.1145/1031607.1031713

[20] DIY "Subway dress" helps protect personal space. TODAY.com. Retrieved August 30, 2018 from http://www.today.com/health/diy-subway-dress-helpsprotect-personal-space-2D79691021

[21] 2016. DIY: SUPERHERO COMMUNICATOR CUFFS. OCAD UNIVERSITY. Retrieved January 2, 2018 from

https://www2.ocadu.ca/research/socialbody/project/di y-superhero-communicator-cuffs

[22] 2018. Drug Relief®. DyAnsys. Retrieved July 18, 2018 from https:/www.dyansys.com/productsapplications/products/drug-relief

[23] L. E. Dunne, H. Profita, C. Zeagler, J. Clawson, S. Gilliland, E. Y. L. Do, and J. Budd. 2014. The social comfort of wearable technology and gestural interaction. In 2014 36th Annual International Conference of the IEEE Engineering in Medicine and Biology Society, 4159-4162. https://doi.org/10.1109/EMBC.2014.6944540

[24] 2018. E-Textiles 2018-2028: Technologies, Markets and Players. IDTechEx. Retrieved August 28, 2018 from https:/www.idtechex.com/research/reports/etextiles-2018-2028-technologies-markets-and-players000613.asp

[25] https://ethicalos.org/. Retrieved September 2, 2018 from https://ethicalos.org/

[26] Event Horizon. Event Horizon. Retrieved September 14, 2018 from http:/www.eventhorizonlarp.com/

[27] Expressive Wearable - by sangli li / Core77 Design Awards. Core 77. Retrieved January 17, 2019 from https://designawards.core77.com/home/award_permali $\mathrm{nk}$ ?id=33483\&category_id=7

[28] Behnaz Farahi. 2016. Caress of the Gaze: A Gaze Actuated 3D Printed Body Architecture. In ACADIA // 2016: POSTHUMAN FRONTIERS: Data, Designers, and Cognitive Machines [Proceedings of the 36th Annual Conference of the Association for Computer Aided Design in Architecture (ACADIA) ISBN 978-0692-77095-5] Ann Arbor 27-29 October, 2016, pp. 352-361. Retrieved September 16, 2018 from http://papers.cumincad.org/cgi-bin/works/paper/acadia 16_352

[29] 2016. Fashion Metamorphosis: Meet the Butterfly Dress. $i Q$ by Intel. Retrieved June 17, 2018 from https://iq.intel.com/fashion-metamorphosis-meet-thebutterfly-dress/

[30] Fitbit Official Site for Activity Trackers \& More. Retrieved August 28, 2018 from 
https://www.fitbit.com/home

[31] Kathleen Flood. 2014. Designing Flying Dresses And The Future Of Fashion, Meet Studio XO. Creators. Retrieved August 29, 2018 from https://creators.vice.com/en_uk/article/jpvq4y/designi ng-flying-dresses-and-the-future-of-fashion-meetstudio-xo-video

[32] Christopher Frayling. 1994. Research in Art and Design (Royal College of Art Research Papers, Vol 1, No 1, 1993/4). Retrieved September 16, 2018 from http://researchonline.rca.ac.uk/384/

[33] Maribeth Gandy, Paul M. A. Baker, and Clint Zeagler. 2017. Imagining futures: A collaborative policy/device design for wearable computing. Futures 87: 106-121. https://doi.org/10.1016/j.futures.2016.11.004

[34] ying gao - designer. Retrieved August 30, 2018 from http://yinggao.ca/interactifs/projets-interactifs/

[35] William W. Gaver. 1991. Technology Affordances. In Proceedings of the SIGCHI Conference on Human Factors in Computing Systems (CHI '91), 79-84. https://doi.org/10.1145/108844.108856

[36] Bill Gaver and John Bowers. 2012. Annotated Portfolios. interactions 19, 4: 40-49. https://doi.org/10.1145/2212877.2212889

[37] William Gaver. 2012. What Should We Expect from Research Through Design? In Proceedings of the SIGCHI Conference on Human Factors in Computing Systems (CHI '12), 937-946. https://doi.org/10.1145/2207676.2208538

[38] James J. Gibson. 2014. The Ecological Approach to Visual Perception: Classic Edition. Psychology Press.

[39] Kiryong Ha, Zhuo Chen, Wenlu Hu, Wolfgang Richter, Padmanabhan Pillai, and Mahadev Satyanarayanan. 2014. Towards Wearable Cognitive Assistance. In Proceedings of the 12th Annual International Conference on Mobile Systems, Applications, and Services (MobiSys '14), 68-81. https://doi.org/10.1145/2594368.2594383

[40] Séverine Halimi-Falkowicz and David Vaidis. 2008. Increasing compliance with a request: two touches are more effective than one. Retrieved July 19, 2018 from https://www.academia.edu/177224/Increasing_compli ance_with_a_request_two_touches_are_more_effectiv e_than_one

[41] Lars Hallnäs and Johan Redström. 2001. Slow Technology - Designing for Reflection. Personal and Ubiquitous Computing 5, 3: 201-212. https://doi.org/10.1007/PL00000019

[42] Kate Hartman, Jackson McConnell, Boris
Kourtoukov, Hillary Predko, and Izzie ColpittsCampbell. 2015. Monarch: Self-Expression Through Wearable Kinetic Textiles. In Proceedings of the Ninth International Conference on Tangible, Embedded, and Embodied Interaction (TEI '15), 413414. https://doi.org/10.1145/2677199.2690875

[43] Marc Hassenzahl and John Carroll. 2010. Experience Design: Technology for All the Right Reasons. Morgan and Claypool Publishers, San Rafael, Calif.

[44] Dan Howarth. 2014. Movie: Studio XO Director explains Lady Gaga's flying dress Volantis. Dezeen. Retrieved August 30, 2018 from https://www.dezeen.com/2014/04/14/movie-studioxo-lady-gaga-flying-dress-volantis/

[45] Katherine Isbister, Kaho Abe, and Michael Karlesky. 2017. Interdependent Wearables (for Play): A Strong Concept for Design. In Proceedings of the 2017 CHI Conference on Human Factors in Computing Systems (CHI'17), 465-471. https://doi.org/10.1145/3025453.3025939

[46] Katherine Isbister, Elena Márquez Segura, Suzanne Kirkpatrick, Xiaofeng Chen, Syed Salahuddin, Gang Cao, and Raybit Tang. 2016. Yamove! A movement synchrony game that choreographs social interaction. Human Technology: An Interdisciplinary Journal on Humans in ICT Environments 12, 1: 74-102. http://dx.doi.org/10.17011/ht/urn.201605192621

[47] Katherine Isbister, Elena Márquez Segura, and Edward F. Melcer. 2018. Social Affordances at Play: Game Design Toward Socio-Technical Innovation. In Proceedings of the 2018 CHI Conference on Human Factors in Computing Systems (CHI '18), 372:1372:10. https://doi.org/10.1145/3173574.3173946

[48] ISWC 2011 - Design Exhibition. Retrieved August 30, 2018 from http://www.iswc.net/iswc11/calls/design_exhibition.html

[49] Hsin-Liu (Cindy) Kao, Deborah Ajilo, Oksana Anilionyte, Artem Dementyev, Inrak Choi, Sean Follmer, and Chris Schmandt. 2017. Exploring Interactions and Perceptions of Kinetic Wearables. In Proceedings of the 2017 Conference on Designing Interactive Systems (DIS '17), 391-396. https://doi.org/10.1145/3064663.3064686

[50] Hsin-Liu (Cindy) Kao, Artem Dementyev, Joseph A. Paradiso, and Chris Schmandt. 2015. NailO: Fingernails As an Input Surface. In Proceedings of the 33rd Annual ACM Conference on Human Factors in Computing Systems (CHI '15), 3015-3018. https://doi.org/10.1145/2702123.2702572 
[51] Hsin-Liu (Cindy) Kao, Christian Holz, Asta Roseway, Andres Calvo, and Chris Schmandt. 2016. DuoSkin: Rapidly Prototyping On-skin User Interfaces Using Skin-friendly Materials. In Proceedings of the 2016 ACM International Symposium on Wearable Computers (ISWC'16), 16-23.

https://doi.org/10.1145/2971763.2971777

[52] Dhruv Khullar. 2016. How Social Isolation Is Killing Us. The New York Times. Retrieved December 12, 2017 from

https://www.nytimes.com/2016/12/22/upshot/howsocial-isolation-is-killing-us.html

[53] Taemie Kim, Agnes Chang, Lindsey Holland, and Alex (Sandy) Pentland. 2008. Meeting Mediator: Enhancing Group Collaboration with Sociometric Feedback. In CHI '08 Extended Abstracts on Human Factors in Computing Systems (CHI EA '08), 31833188. https://doi.org/10.1145/1358628.1358828

[54] Xin Liu, Katia Vega, Pattie Maes, and Joe A. Paradiso. 2016. Wearability Factors for Skin Interfaces. In Proceedings of the 7th Augmented Human International Conference 2016 (AH '16), 21:1-21:8. https://doi.org/10.1145/2875194.2875248

[55] Jonas Löwgren. 2013. Annotated Portfolios and Other Forms of Intermediate-level Knowledge. interactions 20, 1:30-34.

https://doi.org/10.1145/2405716.2405725

[56] Andreas Lymberis and Rita Paradiso. 2008. Smart Fabrics and Interactive Textile Enabling Wearable Personal Applications: R\&D State of the Art and Future Challenges. Conference proceedings : ... Annual International Conference of the IEEE Engineering in Medicine and Biology Society. IEEE Engineering in Medicine and Biology Society. Conference 2008: 5270-3. https://doi.org/10.1109/IEMBS.2008.4650403

[57] Diana MacLean, Asta Roseway, and Mary Czerwinski. 2013. MoodWings: A Wearable Biofeedback Device for Real-time Stress Intervention. In Proceedings of the 6th International Conference on PErvasive Technologies Related to Assistive Environments (PETRA '13), 66:1-66:8. https://doi.org/10.1145/2504335.2504406

[58] Elena Márquez Segura, James Fey, Ella Dagan, Samvid Niravbhai Jhaveri, Jared Pettitt, Miguel Flores, and Katherine Isbister. 2018. Designing Future Social Wearables with Live Action Role Play (Larp) Designers. In Proceedings of the 2018 CHI Conference on Human Factors in Computing Systems (CHI '18), 462:1-462:14. https://doi.org/10.1145/3173574.3174036
[59] Massage me. Retrieved August 30, 2018 from http://www.massage-me.at/

[60] METER. METER. Retrieved August 30, 2018 from https://meter.wearablemedia.studio/

[61] Robb Mitchell. 2015. Sensing Mine, Yours, Theirs, and Ours: Interpersonal Ubiquitous Interactions. In Adjunct Proceedings of the 2015 ACM International Joint Conference on Pervasive and Ubiquitous Computing and Proceedings of the 2015 ACM International Symposium on Wearable Computers (UbiComp/ISWC'15 Adjunct), 933-938. https://doi.org/10.1145/2800835.2806203

[62] Robb Mitchell and Laurens Boer. 2017. Move Closer: Towards Design Patterns To Support Initiating Social Encounters. In Proceedings of the 2017 CHI Conference Extended Abstracts on Human Factors in Computing Systems (CHI EA '17), 2781-2787. https:// doi.org/10.1145/3027063.3053230

[63] Monarch. Vimeo. Retrieved August 30, 2018 from https://vimeo.com/89654806

[64] Jane C. Nannberg and Christine H. Hansen. 1994. Post-Compliance Touch: An Incentive for Task Performance. The Journal of Social Psychology 134, 3: 301-307. https://doi.org/10.1080/00224545.1994.9711734

[65] Todd D. Nelson (ed.). 2017. Getting Grounded in Social Psychology: The Essential Literature for Beginning Researchers. Psychology Press, New York.

[66] My Nguyen. 2017. Wearables for Diabetics. Wearable Technologies. Retrieved September 5, 2018 from https://www.wearable-technologies.com/2017/01/wear ables-for-diabetic/

[67] Don Norman. Don Norman on Wearable Devices. MIT Technology Review. Retrieved July 31, 2018 from https:/www.technologyreview.com/s/517346/theparadox-of-wearable-technologies/

[68] Nudgeables « Kate Hartman. Retrieved August 30, 2018 from http://www.katehartman.com/projects/nudgeables/

[69] Alex "Sandy" Pentland. 2012. The New Science of Building Great Teams. Harvard Business Review. Retrieved September 15, 2018 from https:/hbr.org/2012/04/the-new-science-of-buildinggreat-teams

[70] pixelpeppy - Hug Jackets - pixelpeppy - Despina Papadopoulos. Retrieved October 19, 2017 from https://pixelpeppy.com/hugjackets/

[71] Portfolio. POPKALAB. Retrieved September 16, 2018 from http://www.popkalab.com/portfolio/ 
[72] The Power of Touch. Psychology Today. Retrieved September 4, 2018 from http://www.psychologytoday.com/articles/201303/thepower-touch

[73] Angelica Pursley CNN. 2016. Hussein Chalayan's brand of innovation. CNN Style. Retrieved August 29, 2018 from https:/www.cnn.com/style/article/husseinchalayan-innovation-and-the-fashion-industry/ index.html

[74] Lee Rainie and Kathryn Zickuhr. 2015. Americans' Views on Mobile Etiquette. Pew Research Center: Internet, Science \& Tech. Retrieved September 4, 2018 from http://www.pewinternet.org/2015/08/26/americansviews-on-mobile-etiquette/

[75] Stuart Reeves, Steve Benford, Claire O'Malley, and Mike Fraser. 2005. Designing the Spectator Experience. In Proceedings of the SIGCHI Conference on Human Factors in Computing Systems (CHI '05), 741-750. https://doi.org/10.1145/1054972.1055074

[76] 2010. The Rise of Smart Fashion. The Business of Fashion. Retrieved January 11, 2019 from https://www.businessoffashion.com/articles/intelligen ce/the-rise-of-smart-fashion

[77] 2015. Robotic Spider Dress Powered By Intel Smart Wearable Technology. iQ by Intel. Retrieved August 30, 2018 from https://iq.intel.com/smart-spider-dressby-dutch-designer-anouk-wipprecht/

[78] David A. Ross and Bruce B. Blasch. 2002. Development of a Wearable Computer Orientation System. Personal Ubiquitous Comput. 6, 1: 49-63. https://doi.org/10.1007/s007790200005

[79] Roy Shilkrot, Jochen Huber, Wong Meng Ee, Pattie Maes, and Suranga Chandima Nanayakkara. 2015. FingerReader: A Wearable Device to Explore Printed Text on the Go. In Proceedings of the 33rd Annual ACM Conference on Human Factors in Computing Systems (CHI '15), 2363-2372. https://doi.org/10.1145/2702123.2702421

[80] SmokeBeat ${ }^{\mathrm{TM}}$ smoking cessation monitoring. Somatix. Data with a purpose. Retrieved July 19, 2018 from https://somatix.com/solutions/

[81] 2016. The social age of wearable tech: From Quantified Self to emotional second skin. Wareable. Retrieved August 28, 2018 from https:/www.wareable.com/wearable-tech/the-socialage-of-wearable-tech-beyond-the-quantified-self

[82] Social Body Lab. Social Body Lab. Retrieved January 17, 2019 from http://socialbodylab.com/
[83] Space Dress. Retrieved August 30, 2018 from http:/www.banhomaria.net/spacedress.html

[84] Jacques Terken and Janienke Sturm. 2010. Multimodal Support for Social Dynamics in Colocated Meetings. Personal Ubiquitous Comput. 14, 8: 703-714. https://doi.org/10.1007/s00779-010-0284-x

[85] Oscar Tomico, Lars Hallnäs, Rung-Huei Liang, and Stephan Wensveen. 2017. Towards a Next Wave of Wearable and Fashionable Interactions. International Journal of Design (Online) 11:3.

http://www.ijdesign.org/index.php/IJDesign/article/vie $\mathrm{w} / 3177 / 794$

[86] Watch - Apple. Retrieved August 28, 2018 from https://www.apple.com/watch/

[87] Wearable Technology Future is Ripe for Growth Most Notably among Millennials, Says PwC US. Retrieved September 4, 2018 from https://www.prnewswire.com/news-releases/wearabletechnology-future-is-ripe-for-growth--most-notablyamong-millennials-says-pwc-us-515861911.html

[88] 2017. Why is Sweden's "lagom" leadership taking the world by storm? Retrieved December 3, 2017 from https://www.thelocal.se/20171127/why-is-swedenslagom-leadership-taking-the-world-by-stormsseexecutiveeducation-tlccu

[89] Frank N. Willis and Helen K. Hamm. 1980. The use of interpersonal touch in securing compliance. Journal of Nonverbal Behavior 5, 1: 49-55. https://doi.org/10.1007/BF00987054

[90] George M. Wyburn, Ralph W. Pickford, and R. J. Hirst. 1964. Human Senses And Perception. University Of Toronto Press.

[91] XO. Retrieved January 17, 2019 from http://www.studio-xo.com/

[92] Yang Claire Yang, Courtney Boen, Karen Gerken, Ting Li, Kristen Schorpp, and Kathleen Mullan Harris. 2016. Social relationships and physiological determinants of longevity across the human life span. Proceedings of the National Academy of Sciences 113, 3: 578-583. https://doi.org/10.1073/pnas.1511085112

[93] Clint Zeagler. 2017. Where to Wear It: Functional, Technical, and Social Considerations in On-body Location for Wearable Technology 20 Years of Designing for Wearability. In Proceedings of the 2017 ACM International Symposium on Wearable Computers (ISWC'17), 150-157. https://doi.org/10.1145/3123021.3123042

[94] John Zimmerman, Jodi Forlizzi, and Shelley Evenson. 2007. Research Through Design As a Method for Interaction Design Research in HCI. In Proceedings of the SIGCHI Conference on Human Factors in 
Computing Systems (CHI '07), 493-502. https://doi.org/10.1145/1240624.1240704

[95] John Zimmerman, Erik Stolterman, and Jodi Forlizzi. 2010. An Analysis and Critique of Research Through
Design: Towards a Formalization of a Research Approach. In Proceedings of the 8th ACM Conference on Designing Interactive Systems (DIS '10), 310-319. https://doi.org/10.1145/1858171.1858228 